DEMONSTRATIO MATHEMATICA

Vol. XVIII

No 2

1985

Teresa Markiewicz, Lucyna Rempulska

\title{
ON THE APPLICATION OF THE LEGENDRE POLYNOMIALS TO THE DIRICHLET PROBLEM FOR THE LAPLACE EQUATION
}

1. Iet $r, \theta, t$ be the spherical coordinates of points,
$Q=\{(r, 8, t): 0<r<1,0 \leqslant \theta \leqslant \pi, 0 \leqslant t \leqslant 2 \pi\}, S=\{(r, 8, t)$ : $r=1,0 \leqslant 8 \leqslant \pi, 0 \leqslant t \leqslant 2 \pi\}$ and $\bar{Q}=Q+S$. Let $R$ be the rectangle defined by $0 \leqslant 6 \leqslant \pi, 0 \leqslant t \leqslant 2 \pi$.

Denote by $C^{m}(Q)$ (m is a non-negative integer, 1.e. m $\in \mathbb{N}$ ) the clase of all real-valued functiong defined in $Q$ and having the partial derivatives of the order $\leqslant m$ continuous in $Q$. Analogousis: will be interpreted the symbols $C^{m}(\bar{Q})$ and $C^{\infty}(Q)$.

The ejmbol $C^{m}(R)$ will denote the class of functions having the properties as above and euch that $f(\theta+\pi, t+2 \pi)=f(s, t)$.

Let $\Delta$ be the Laplace operator, i.e. $\Delta=\frac{\partial^{2}}{\partial r^{2}}+\frac{2}{r} \frac{\partial}{\partial r}+$ $+\frac{1}{r^{2}} \frac{\partial^{2}}{\partial s^{2}}+\frac{\cos \theta}{r^{2} \sin s} \frac{\partial}{\partial s}+\frac{1}{r^{2} \sin ^{2} s} \frac{\partial^{2}}{\partial t^{2}}$, and $\Delta^{n} u=\Delta\left(\Delta^{n-1} u\right)$ for $n=2,3, \ldots$ and $u \in C^{\infty}(Q),\left(\Delta^{1} \equiv \Delta\right)$.

In this paper we shall give a solution of the Lirichlet problem for the equetion $\Delta^{n} u(r, s, t)=0$ in $\vec{Q}$. We shall construct the function $u$ of the class $c^{n}(\bar{Q}), n \geqslant 1$, such that $\Delta^{n+1} u(r, s, t)=0$ in $Q,\left.u(r, s, t)\right|_{S}=f(s, t)$, where $f$ is $a$ fixed function of the class $C(R)\left(C(R)=C^{0}(R)\right)$, and $\left.\frac{\partial p_{u}}{\partial r}\right|_{S}=0$ for $p=1, \ldots, n$.

The solution of Dirichlet's problem for $\Delta u(r, s, t)=0$ in $\bar{Q}$ was given in [3] (p.472).

The similar problem was congidered in [2]. 
2. Uaing the mathematioal induction, we oan prove

I $m a n$ a If $u \in c^{\infty}(Q)$ and $n=1,2, \ldots$, then

$$
\begin{aligned}
\Delta^{n}\left(r \frac{\partial u}{\partial r}\right) & =r \frac{\partial}{\partial r} \Delta^{n} u(r, \theta, t)+2 n \Delta^{n} u(r, \theta, t) ; \\
\Delta^{n}\left(r^{3} \frac{\partial u}{\partial r}\right) & =r \frac{\partial}{\partial r} \Delta^{n-1}\left(6 u(r, \theta, t)+r^{2} \Delta u(r, 8, t)+4 r \frac{\partial u}{\partial r}\right)+ \\
& +2(n-1) \Delta^{n-1}\left(6 u(r, 8, t)+r^{2} \Delta u(r, \theta, t)+4 r \frac{\partial u}{\partial r}\right)
\end{aligned}
$$

and

$$
\begin{aligned}
\Delta^{n}\left(r^{2} \Delta u(r, 8, t)\right) & =r^{2} \Delta^{n+1} u(r, 8, t)+4 n r \frac{\partial}{\partial r} \Delta^{n} u(r, 8, t)+ \\
& +(6 n+4 n(n-1)) \Delta^{n} u(r, 8, t) \text { for }(r, 8, t) \in Q_{.}
\end{aligned}
$$

From Lemma 1 and by the linearity of the operator $\Delta^{n}$ we obtain

I $\theta m a$ 2. If $u \in c^{2 n+2}(Q)(n \geqslant 1)$ and $\Delta^{n} u(r, s, t)=0$ in $Q$, then the function $\nabla$,

$$
v(r, s, t)=u(r, s, t)+\frac{r-r^{3}}{2(n+1)} \frac{\partial}{\partial r} u(r, s, t),
$$

satisfies the equation $\Delta^{n+1} v(r, a, t)=0$ in $Q$.

Moreover, the following result can be easils obtained.

I $e \operatorname{mm} a$ 3. If $u \in C^{n+2}(\bar{Q}), n \geqslant 1$, and $\left.\frac{\partial^{p} u}{\partial r p}\right|_{S}=0$ for

$p=1, \ldots, n$, then $v$ defined by (1) satisfies the condition $\left.\frac{\partial^{p}}{\partial r}\right|_{S}=0$, with $p=1, \ldots, n+1$.

3. Let $a_{k, I}(f), b_{k, I}(f), c_{k, I}(f)$ and $d_{k, I}(f)$ be the coefficients of doubie trigonometric Fourier series of $f \in C(R)$.

L e m m 4. (cf. [4], [5]). If $f \in C^{2 n+2}(R)(n \geqslant 0)$, then, for $\theta$ very $p, q \in \mathbb{N}$ and $p+q=2 n$, the series 
$\sum_{k=0}^{\infty} \sum_{I=0}^{\infty}(k+1)^{p}(I+1)^{q}\left(\left|a_{k, I}(f)\right|+\left|b_{k, I}(f)\right|+\left|c_{k, I}(f)\right|+\left|a_{k, I}(f)\right|\right)$
I. oonvergent ${ }^{*}$.

4. Let $P_{n}$ be the Legendre polgnomial

$$
P_{n}(x)=\frac{1}{2^{n} n !} \frac{d^{n}}{d x^{n}}\left(x^{2}-1\right)^{n} \quad(n=0,1, \ldots ; x \in<-1,1>),
$$

and let

$$
F_{n, m}(x)=\left(1-x^{2}\right)^{\frac{m}{2}} \frac{d^{m}}{d x^{m}} P_{n}(x)
$$

for $x \in\langle-1,1\rangle, n=1,2, \ldots$ and $m=1, \ldots, n([3], p .453)$.

Denote by $A_{k, l}(f)$ and $B_{k, l}(f)$ the coefficients of the Fourier series of $f \in C(R)$ with the orthogonal system

i.8.

$$
P_{k, I}(\cos s)\left(\begin{array}{ll}
\cos 1 t \\
\sin 1 t
\end{array}\right)((s, t) \in R)
$$

$$
\begin{aligned}
& A_{k, 0}(f)=\frac{2 k+1}{4 \pi} \iint_{R} f(s, t) P_{k}(\cos s) \sin s d s d t \\
& A_{k, I}(f)=\frac{(2 k+1)(k-1) !}{2 \pi(k+1) !} \iint_{R} f(s, t) P_{k, I}(\cos s) \cos 1 t \sin s d s d t, \\
& B_{k, I}(f)=\frac{(2 k+1)(k-1) !}{2 \pi(k+1) !} \iint_{R} f(s, t) P_{k, I}(\cos s) \sin 1 t \sin s d s d t \\
& ([3], \mathrm{p} .455) \text {. Let, as in [3], } \\
& \text { *) We shall say that a series } \sum_{k=0}^{\infty} \sum_{l=0}^{\infty} \jmath_{k, l} \text { is convergent } \\
& \text { if there exists } \lim _{m, n \rightarrow \infty} \sum_{k=0}^{m} \sum_{l=0}^{n} y_{k, 1}=\alpha \text { and }|\alpha|<+\infty \text {. }
\end{aligned}
$$




$$
\begin{gathered}
Y_{0}(s, t ; f)=A_{0,0}(f), \\
Y_{k}(s, t ; f)=A_{k, 0}(f) P_{k}(\cos \theta)+ \\
+\sum_{I=1}^{K}\left(A_{k, I}(f) \cos I t+B_{k, I}(f) \sin 1 t\right) P_{k, I}(008 \theta)
\end{gathered}
$$

for $k=1,2, \ldots ;(s, t) \in R$ and $f \in C(R)$. In [3] (p.467) there was given a jufficient condition for the convergonoe to $f$ of the Fourier series

$$
\sum_{k=0}^{\infty} Y_{k}(s, t ; f) \quad((s, t) \in R)
$$

It is clear that $P_{n}(\cos 8)$ and $P_{n, m}(00 \theta 8)$ are some trigo-. nometric polynomials of the order $n$. The series (2) can be written in the form of double trigonometric Fourier series for the function $f$ if it is absolutels convergent in $R$. Lemme 4 and the result given in [3] (p.467) imply the following Ierrmas:

I $\theta$ in a 5. If $f \in C^{2}(R)$, then the series (2) is convergent to $f$ uniformly. Moreover, this series is absolutely convergent for every $(s, t) \in R$.

I $\theta \mathrm{m}$ a 6. Suppose that $f \in \mathrm{C}^{2 n+2}(R)(n \geqslant 1), p, q \in \mathbb{N}$ and $p+q=2 n$. Then the series

$$
\sum_{k=0}^{\infty}(k+1)^{2 n} Y_{k}(s, t ; f) \text { and } \sum_{k=0}^{\infty} \frac{\partial^{2 n}}{\partial s^{p} \partial t^{q}} Y_{k}(s, t ; f)
$$

are absolutely convergent for every $(s, t) \in R$.

From Lemmas 5, 6 and by the fundamental theorems on power series there follows:

Cor o I I a $y$ 1. If $f \in C^{2 n+2}(R) \geqslant(n \geqslant 0)$, then $\mathrm{U}_{\mathrm{O}}(\mathrm{f})$, defined by forcula 


$$
U_{0}(r, \theta, t ; f)=\sum_{k=0}^{\infty} r^{k} Y_{k}(\theta, t ; f)
$$

is a function of the class $c^{2 n}(\bar{Q})$. Moreover, $U_{U}(f) \in C^{\infty}(Q)$ and

$$
U_{0}(1,8, t ; f)=f(s, t) \quad((s, t) \in R)
$$

5. Iet, as in $[1]$ :

(4) $D^{n}\left(r^{k}\right)=\left\{\begin{array}{l}r^{k} \quad \text { if } n=0, \\ D^{n-1}\left(r^{k}\right)+\frac{r-r^{3}}{2 n} \frac{d}{d r} D^{n-1}\left(r^{k}\right) \text { if } n=1,2, \ldots\end{array}\right.$

for $k=0,1, \ldots$ and $r \in\langle 0,1\rangle$.

By mathematical induction we can prove

I $\circ \mathrm{m}$ a 7. If $n=1,2, \ldots$, then

$$
D^{n}\left(r^{k}\right)=r^{k}+\sum_{q=1}^{n} W_{q}(r ; n) \frac{d^{q}}{d r^{q}} r^{k} \quad(k=0,1, \ldots ; r \in\langle 0,1>),
$$

where $W_{q}$ are some algebraic polynomiale with coefficients depending on $n$ only and such that

$$
\left(\frac{d^{p}}{d r^{p}} W_{q}(r ; n)\right)_{r=1}=\left\{\begin{array}{cl}
0 & \text { if } p \neq q \\
(-1)^{p} & \text { if } p=q
\end{array}\right.
$$

for $p=0,1, \ldots, n$ and $q=1, \ldots, n \quad(s \theta e \quad[1])$.

Arguing aimilarly as in [1], [2], we shall prove

Th. $\theta \circ r \in m$. Suppose that $f \in c^{2 n+2}(R), n \geqslant 0$, and $Y_{k}(f), D^{n}\left(x^{k}\right)$ are defined as in (2) and (4). Then the function

$$
U_{n}(f)=U_{n}(r, a, t ; f)=\sum_{k=0}^{\infty} D^{n}\left(r^{k}\right) Y_{k}(a, t ; f)
$$


has the properties:

$$
\begin{array}{ll}
1^{0} & U_{n}(f) \in C^{n}(\bar{Q}), \\
2^{0} & \Delta^{n+1} U_{n}(r, s, t ; f)=0 \text { for }(r, s, t) \in Q, \\
3^{0} & \left.U_{n}(r, s, t ; f)\right|_{S}=f(s, t)
\end{array}
$$

and, if $n \geqslant 1$,

$\left.4^{0} \frac{\partial^{p}}{\partial r^{p}} U_{n}(r, \theta, t ; f)\right|_{S}=0$, for $p=1, \ldots, n$.

P r o o $f$. The conditions $1^{\circ}-3^{0}$ for $U_{0}(f)$ are given in [3] $(0.455-472)$.

By Corollary 1, Lemma 7 and (4), (5), we obtain

(6) $U_{m}(r, s, t ; f)=U_{0}(r, s, t ; f)+\sum_{q=1}^{m} W_{q}(r ; m) \frac{\partial^{q}}{\partial r^{q}} U_{0}(r, s, t ; f)$

for $(r, \theta, t) \in \bar{Q}$ and $1 \leqslant m \leqslant n$. Moreover,

(7) $U_{m}(r, s, t ; f)=U_{m-1}(r, s, t ; f)+\frac{r-r^{3}}{2 r} \frac{\partial}{\partial r} U_{m-1}(r, s, t ; f)$

for $(r, s, t) \in \bar{Q}$ and $1 \leqslant m \leqslant n$.

Hence, by (6) and Corollary 1, we get

$$
U_{m}(f) \in c^{\infty}(Q) \text { and } U_{m}(f) \in c^{2 n-m}(\bar{Q})
$$

for $0 \leqslant m \leqslant n$.

The condition $2^{\circ}$ for $U_{0}(f),(7)-(8)$ and Lemme 2 imply $2^{\circ}$ for $U_{n}(f)$. The condition $3^{\circ}$ holds by $(3)-(5)$.

If $n \geqslant 1$, then, by $(7)$ and $(8)$,

$$
\left.\frac{\partial}{\partial r} U_{1}(r, \boldsymbol{r}, t ; f)\right|_{S}=0
$$

Applying (7) $-(9)$ and Lemma 3, we obtain $4^{\circ}$ for $U_{n}(f)$. Thue the proof is completed. 
REFERENCES

[1] Z. D D pioraza, L. $R \theta m p u l s k a:$ on the summability of series by harmonic methods, Comment. Math. Praoe Mat. 23, 2 (1983), 11-25.

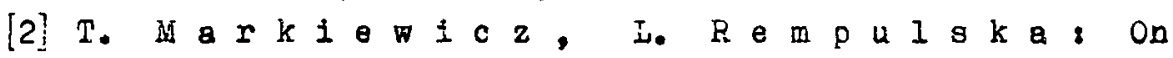
the application of the Fourier-Iegerdre serieg for the Laplace type, Comment. Math. Prace Mat. (in print).

[3] W.I. S m i r n ow: Matematyka myzaza, T.3 (2), Warszawa, 1967.

[4] М.Ф. Т и м а н : 06 абсолотной сходиности кратнах рядов Фypbe, Dokl. Akad. Nauk SSSR, 137 (1961), 1074-1077.

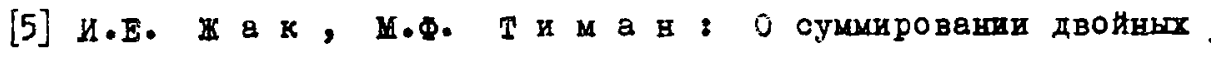
pндОв, Mat. Sb., $35(1954), 1,21-56$.

INSTITUTE OF MATHEMATICS, A.BICKIEWICZ UNIVERSITY,

60-769 POZNAN, POLAND;

INSTITUTE OF MATHEMATICS, TECHNICAL UNIVERSITY OF POZNAN, 60-965 POZNAN, POLAND

Received February 3, 1984. 
